\title{
MICROPOLUENTES EM ÁGUA - O NOVO DESAFIO EMERGENTE
}

\author{
Gabriela Vettorello ${ }^{1}$, Verônica Brandt ${ }^{2}$, Maria Cristina Dallazen ${ }^{3}$, Daniel Kunh ${ }^{4}$, \\ Henrique Pretto Etgeton ${ }^{5}$, Julia Grasiela Spellmeyer ${ }^{6}$, Wagner Manica Carlesso, \\ Lucélia Hoehne ${ }^{8}$
}

\begin{abstract}
Resumo: O interesse pela análise de fármacos e outros micropoluentes em água vem ganhando destaque ao longo dos anos com o aprimoramento das técnicas analíticas e equipamentos sofisticados capazes de detectar substâncias em concentrações mínimas. A atual legislação nacional estipula valores máximos para agrotóxicos, desinfetantes e outros componentes orgânicos e inorgânicos. Porém, o atual tratamento convencional da água de abastecimento não visa à eliminação de antibióticos e demais fármacos, que consequentemente não são abordados nos limites padrões de potabilidade. Parte da concentração dos medicamentos ingeridos são excretados e destinados ao esgoto doméstico que, após tratamento, retornam à fonte mantenedora da água de abastecimento da população. $\mathrm{O}$ presente trabalho tem por objetivo fundamentar teoricamente por meio de uma revisão bibliográfica, baseada na consulta a artigos científicos selecionados através do banco de dados da Elsevier, um estudo enfatizando micropoluentes, mais precisamente, fármacos da classe dos antibióticos. Concluiu-se que técnicas diferenciadas de preparação de amostras e uso da Cromatografia Líquida são as mais adequadas e indicadas para detectar e quantificar estes componentes, capazes de atingir níveis de limites de detecção na faixa $\mu \mathrm{g} / \mathrm{L}$ e que a determinação de micropoluentes é uma área em ascensão, devido à sua complexidade e aos efeitos de sua assimilação no organismo humano serem ainda desconhecidos.
\end{abstract}

Palavras-chave: Micropoluentes. Fármacos. Cromatografia. Água. Efluente.

1 Graduanda em Química Industrial - Univates - gvettorello@univates.br

2 Graduanda em Química Industrial - Univates- vvbrandt@universo.univates.br

3 Química Industrial - Univates - crisdallazen@yahoo.com.br

4 Química Industrial - Mestrando em Biotecnologia - Univates - danielkuhn@universo.univates.br

5 Graduando em Engenharia Ambiental - Univates - henrique.etgeton@universo.univates.br

6 Química Industrial, Mestre em Química - Univates - jgs@univates.br

7 Engenheiro Ambiental - Univates - wmcarlesso@univates.br

8 Química Industrial, Doutora em Química, PPGBiotecnologia - Univates - luceliah@univates.br 


\title{
MICROPOLUENTES IN WATER - THE NEW \\ EMERGING CHALLENGE
}

\begin{abstract}
The interest in analyzing drugs and other micropollutants in water has been gaining prominence over the years with the improvement of analytical techniques, which are now able to detect substances even at trace levels. Current national legislation stipulates maximum values for pesticides, disinfectants and other organic and inorganic components, but the current conventional treatment of water supply does not aim the elimination of antibiotics, which consequently are not approached within the standard limits of potability. The consumption of drugs causes the excretion of part of the concentration of the same, which are destined to the domestic sewage and after treatment, return to the source of the supply water for the population. The objective of this study is to provide a theoretical basis for the subject of micropollutants, emphasizing antibiotic drugs, and the research methodologies used to identify them, through a bibliographic review based on the consultation of scientific articles selected through the database of the Elsevier. It was concluded that Liquid Chromatography analyzes are the most adequate and indicated to detect and quantify these components, being capable of reaching levels of detection limits in the $\mu \mathrm{g} / \mathrm{L}$ range and that the determination of micropollutants is a growing area due to its complexity and the effects of their assimilation on the human organism are still unknown.
\end{abstract}

Keywords: Micropollutants. Drugs. Chromatography. Water. Efluent.

\section{INTRODUÇÃO}

A água no estado sólido se apresenta como gelo e contribui com $2 \%$ de seu volume total no planeta, enquanto que na forma líquida constitui $98 \%$. Neste estado, ela possui maior utilidade aos organismos vivos, sendo representada $97 \%$ por mares e oceanos e $1 \%$ por águas continentais, superficiais ou subterrâneas, sendo estas utilizadas para consumo (MAGOSSI; BONACELLA, 2008).

Alguns estudos apontam que a média de consumo humano chega a 700 litros de água por dia, e em alguns países mais desenvolvidos da Europa chega a 1.700 litros. Nos Estados Unidos, pode-se chegar a 8.000 litros diários para cada habitante (MAGOSSI; BONACELLA, 2008).

O tratamento da água bruta, no processo para caracterização para água de abastecimento, tem como base razões sanitárias, com a remoção de bactérias, elementos nocivos, mineralização excessiva, teores elevados de compostos orgânicos, protozoários e outros microrganismos, além de razões estéticas que envolvem correção da cor, sabor e turvação (ALVES, 2010).

Existem vários tratamentos convencionais de efluentes baseados em processos físicos, químicos e biológicos. Os processos biológicos, por conseguirem tratar um maior volume de efluente com uma grande eficiência em remoção de matéria orgânica e serem mais baratos, são os mais utilizados (CLARA et al., 2005; RADJENOVIC, 2007). Apesar de alcançar altas taxas de remoção de matéria orgânica, a taxa de biodegradabilidade de fármacos ainda está na ordem de $50 \%$ para sistemas convencionais de lodo ativado (FENT et al., 2006; RADJENOVIC, 2007). Os processos físicos, 
como a decantação, flotação, entre outros, são mais úteis como pré ou pós-tratamento do processo final, pois estes não degradam o contaminante, apenas o transfere de fase (FREIRE et al., 2000; KUNZ et al., 2002). Já os processos químicos baseiam-se na mineralização dos contaminantes a $\mathrm{CO}_{2}$ através da oxidação, que ocorre pela reação com oxidantes fortes. Porém, este tratamento também não é totalmente eficiente, havendo a formação de subprodutos de degradação, normalmente ácidos orgânicos (VELLA; MUNDER, 1993). A ineficiência dos processos convencionais de tratamento de efluente em relação aos micropoluentes requer a utilização de processos alternativos, denominados de processos oxidativos avançados, citados por Dias (2012) em revisão de Andreozzi et al. (1999) que se baseiam na reatividade de radicais hidroxila gerados que, por serem pouco seletivos, podem oxidar uma vasta gama de substâncias.

A qualidade final da água tratada depende das características de sua fonte, pois alguns compostos químicos tóxicos, de origem química ou biológica, dificilmente são removidos pelos tratamentos convencionais (ALVES, 2010).

Tradicionalmente, o foco dos poluentes químicos é quase que exclusivamente nos poluentes mais convencionais como os hidrocarbonetos policíclicos aromáticos (HPAs), bifenilos policlorados (PCBs) e os pesticidas organoclorados. Entretanto, o uso desenfreado de agrotóxicos e o crescente uso de medicamentos geraram um desafio para o estudo da deteç̧ão de micropoluentes emergentes, que se tornou um novo e grave problema ambiental (SATINDER, 2009). Dessa forma, este artigo tem o objetivo de fundamentar teoricamente por meio de uma revisão bibliográfica, um estudo enfatizando micropoluentes, mais precisamente, fármacos da classe dos antibióticos. Para isso, foram feitos levantamentos de artigos baseada na consulta a artigos científicos selecionados através do banco de dados da Elsevier entre março e junho de 2017.

\section{MICROPOLUENTES}

A preocupação com micropoluentes emergentes já vem de longa data. Na década de 1970, nos Estados Unidos, Garrison et al. (1976) e Hignite e Azarnof (1977) realizaram estudos sobre fármacos no meio ambiente, encontrando-os em efluentes de estações de tratamento de esgoto (ETE), ácido clofíbico, metabólito dos antilipêmicos clofibrato e etofibrato, com concentrações em $\mu \mathrm{g} / \mathrm{L}$.

Conforme Vella e Munder (1993), os micropoluentes classificam-se em poluentes orgânicos persistentes (POPs) e micropoluentes orgânicos emergentes. Os POPs podem permanecer por muito tempo no ambiente e se bioacumular em tecidos gordurosos de seres vivos, pelo fato de serem substâncias químicas com resistência a biodegradação, apresentando uma toxicidade aguda e crônica em baixas concentrações (BILA; DEZOTTI, 2007).

Denominam-se de micropoluentes emergentes os contaminantes que só puderam ser detectados e quantificados recentemente por meio do desenvolvimento de novas 
técnicas analíticas sensíveis e mais complexas. Isto deve-se ao fato destes micropoluentes disponibilizarem-se em concentrações muito baixas na água, na faixa de $\mu \mathrm{g} / \mathrm{L}$ e ng/L, com sua toxicidade aquática a organismos terrestres ainda relativamente desconhecida (SATINDER, 2009; BILA; DEZOTTI, 2007).

Com estas características, classificam-se como micropoluentes emergentes os fármacos e os agrotóxicos, amplamente utilizados nas lavouras brasileiras, os aceleradores de vulcanização, os repelentes, hormônios naturais e sintéticos, os compostos químicos presentes em produtos de higiene pessoal, de limpeza, e outros diversos produtos químicos amplamente utilizados hoje em dia pela sociedade, aumentando cada vez mais a sua quantidade no ecossistema aquático (REIS et al., 2007; SOARES; LEÃO, 2015). A Tabela 1 apresenta alguns micropoluentes emergentes em ambientes aquáticos, acompanhados de suas subclasses e suas fontes.

Tabela 1 - Micropoluentes emergentes com suas subclasses e fontes

\begin{tabular}{|c|c|c|}
\hline Categoria & Subclasse & Fonte \\
\hline Fármacos & $\begin{array}{l}\text { Drogas (anti-inflamatórios, } \\
\text { anticonvulsivos, antibióticos, } \\
\text { estimulantes, analgésicos e } \\
\text { outros) }\end{array}$ & $\begin{array}{l}\text { Esgotos domésticos, efluentes } \\
\text { de hospital, escoamento } \\
\text { CAC*, aquicultura }\end{array}$ \\
\hline Produtos de cuidado pessoal & $\begin{array}{l}\text { Fragrâncias, desinfetantes, filtros } \\
\text { solares, repelentes de insetos e } \\
\text { outros }\end{array}$ & Esgotos doméstico \\
\hline Hormônios esteroides & Estrogênios & $\begin{array}{l}\text { Esgoto doméstico, escoamento } \\
\text { CAC }\end{array}$ \\
\hline Surfactantes & Surfactantes não iônicos & $\begin{array}{l}\text { Esgoto doméstico, efluente } \\
\text { industrial }\end{array}$ \\
\hline Químicos industriais & Retardantes de chama & $\begin{array}{l}\text { Esgoto doméstico, efluentes } \\
\text { industriais }\end{array}$ \\
\hline Agrotóxicos & $\begin{array}{l}\text { Inseticidas, herbicidas, fungicidas } \\
\text { e outros }\end{array}$ & $\begin{array}{l}\text { Esgoto doméstico, escoamento } \\
\text { superficial em áreas agrícolas }\end{array}$ \\
\hline
\end{tabular}

Fonte: Luo et al. (2014).

${ }^{*}$ CAC: Criadouros de animais confinados.

A partir das informações citadas, é provavelmente que a água que é considerada potável esteja contaminada por substâncias que ainda não são registradas pela legislação, podendo ser muito nocivas aos ecossistemas aquáticos e à saúde humana (SOARES; LEÃO, 2015).

Como a legislação brasileira vigente se refere apenas a contaminantes detectados e identificados há vários anos, esta não determina limites que sejam seguros para contaminantes emergentes, já que estes foram identificados no ambiente recentemente (BOLONG et al., 2009). Existem estudos voltados à identificação e quantificação destas classes denominadas micropoluentes, mas, ainda são escassas políticas de tratamento adequado para removê-los e que, possam futuramente possibilitar a atualização das 
normas ambientais atualmente disponíveis (BOLONG et al., 2009; MELLO et al., 2009).

A Portaria MS no 2.914 de 12/12/2011 dispõe sobre os procedimentos de controle e de vigilância da qualidade da água para consumo humano e seu padrão de potabilidade. O documento apresenta em seu anexo VII uma tabela de padrão de potabilidade para substâncias químicas que representam risco à saúde, e em seu anexo XII há uma tabela de número mínimo de amostras e frequência para o controle da qualidade da água de sistema de abastecimento, para fins de análises físicas, químicas e de radioatividade, em função do ponto de amostragem, da população abastecida e do tipo de manancial. As informações contidas no documento são omissas a valores máximos permitidos de fármacos em geral, o que indica que estes parâmetros não são tratados e avaliados periodicamente na água tratada para consumo humano.

Mais de 3.000 substâncias químicas são utilizadas na medicina humana e veterinária, incluindo anti-inflamatórios, contraceptivos, beta-bloqueadores, reguladores lipídicos, tranquilizantes, antiepilépticos e antibióticos (SATINDER, 2009).

A era da antibioticoterapia iniciou em 1941, com o isolamento da penicilina por Florey e Chain, que retomaram as pesquisas de Alexander Fleming, descobridor da penicilina $G$, em 1928. Os antibióticos são medicamentos obtidos a partir de determinados microrganismos, vegetais ou sínteses laboratoriais, que combatem infecções de bactérias e outros microrganismos (TAVARES, 2009).

Verlicchi et al. (2009), a partir da revisão de Ternes e Joss (2006), apresenta um consumo anual do antibiótico sulfametoxazol de 22, 4 toneladas na França, 47 toneladas na Alemanha, 12,7 toneladas na Espanha, enquanto que o consumo médio anual de amoxicilina em 2001 era de 110 toneladas na Alemanha e na Itália, e nos Estados Unidos, 23.000 toneladas por ano. Considerando que destes, cerca de $55 \%$ a $80 \%$, com algumas exceções, não são metabolizados, sendo excretados pela urina.

Em outro estudo, realizado na Suécia em 2003, realizou-se a determinação de antibióticos no efluente de um hospital por meio da coleta de amostras realizada periodicamente ao longo de um dia. Utilizando-se da metodologia de extração em fase sólida (Solid Phase Extraction - SPE) e Cromatografia Líquida de alta eficiência (do inglês High Performance Liquid Cromatography - HPLC), os autores quantificaram concentrações na faixa de $100 \mu \mathrm{g} / \mathrm{L}$, principalmente do antibiótico ciprofloxacina, em determinados horários de coleta (LINDBERG et al., 2003).

Verlicchi et al. (2009) concluem que a relação de consumo de água e contribuição de cargas poluentes entre efluentes hospitalares e efluentes urbanos é de 2 a 3 , enquanto que a contribuição de micropoluentes varia no intervalo de 4 a 150 . Os autores recomendam a utilização de tratamentos separados para estes efluentes, a fim de evitar a diluição devido à mistura com os esgotos urbanos. São sugeridos tratamentos biológicos, ultrafiltração com membranas especiais, ozonização, processos oxidativos 
avançados e ainda a utilização de polímeros, mas salientam a importância de outros estudos que avaliem a capacidade de remoção de micropoluentes, com informações mais técnicas e sob um ponto de vista econômico. A Figura 1 apresenta um fluxograma estimado do destino dos fármacos, apresentado por Bila e Dezotti (2003).

Figura 1 - Possíveis rotas de fármacos no meio ambiente

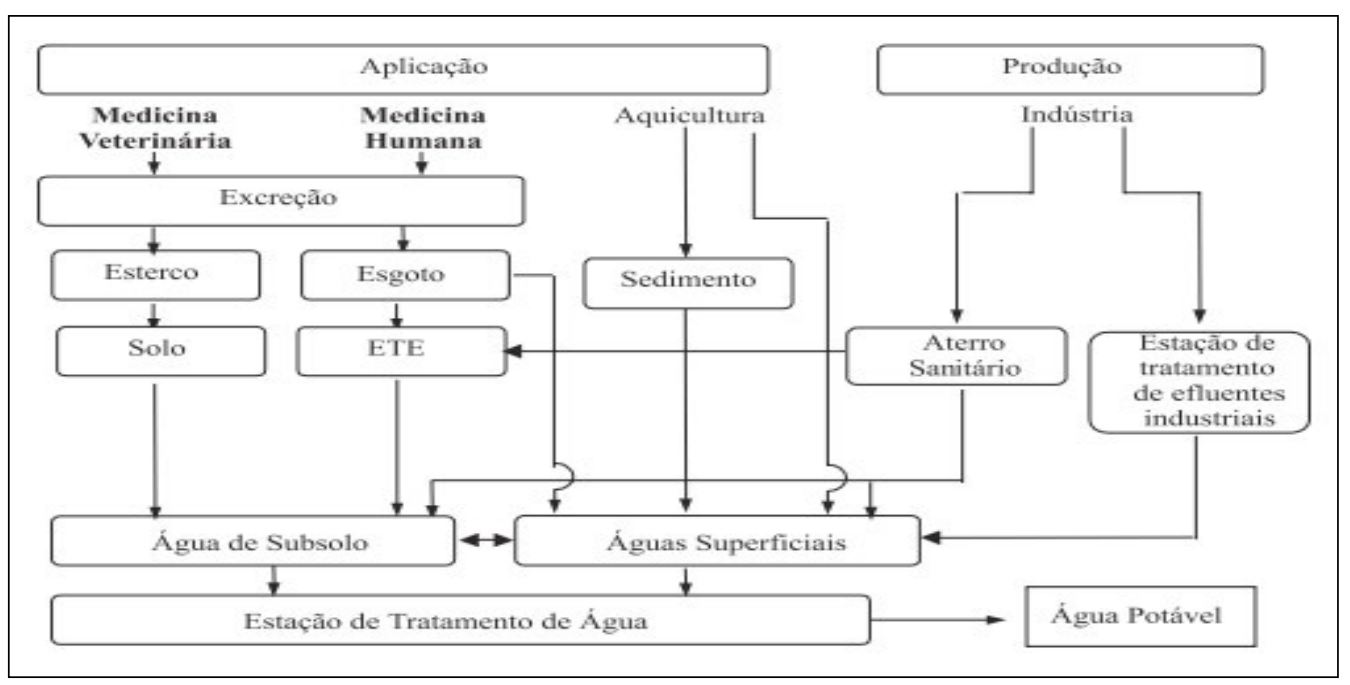

Fonte: Bila e Dezotti (2003).

Em revisão bibliográfica de Kummerer (2001); Petrovic et al. (2003); Carballa et al. (2004); Onesios et al. (2009), Verlicchi et al. (2009) informa que efluentes de hospitais não são a única fonte de fármacos. Resíduos destes micropoluentes são encontrados em águas residuais devido à ineficiência da remoção pelos sistemas convencionais de tratamento. A dificuldade de remoção destes componentes, especialmente fármacos, se deve às baixas concentrações em que se apresentam, na faixa de 10-3 a 10-6 mg/L, muito abaixo dos valores convencionais de macropoluentes como $\mathrm{DBO}_{5}, \mathrm{DQO}$, compostos nitrogenados e fosforados. Além disso, o comportamento destes micropoluentes em estações de tratamento é afetado devido a uma ampla diferença em suas propriedades, como solubilidade, volatilidade, adsorção, absorção, biodegradabilidade, polaridade e estabilidade.

Em seu artigo sobre o Descarte de Medicamentos no Meio Ambiente no Brasil, Souza e Falqueto (2015) apontam a necessidade de um trabalho de conscientização ambiental visando o uso racional de medicamentos e seu correto descarte, citando também a importância da aplicação de uma logística reversa, que visa à devolução de determinados resíduos aos fabricantes de modo que estes insiram dentro do seu ciclo produtivo e possam dar o tratamento e a destinação final adequada.

Alguns micropoluentes são capazes de desregular o sistema endócrino humano e animal, e atualmente questiona-se quanto à exposição a estes componentes: quais 
os efeitos que os mesmos podem produzir em baixas concentrações, quais substâncias associam-se aos efeitos tóxicos em baixas concentrações, se atualmente essas substâncias encontram-se em concentrações relevantes e se existe uma concentração limite em que elas se apresentem que possa ser considerada segura (BILA; DEZOTTI, 2007).

O conhecimento dos níveis em que micropoluentes como substâncias sintéticas (alquilfenóis, pesticidas, ftalatos, policlorados de bifenilas (PCD), bisfenol A, substâncias farmacêuticas, entre outras) e substâncias naturais (estrogênios naturais e fitoestrogênios) se encontram é fundamental para avaliar seus efeitos potenciais no organismo (BILA; DEZOTTI, 2007), justificando a necessidade e importância do desenvolvimento de técnicas avançadas, capazes de detectar e quantificar estes componentes em água potável.

\subsection{Preparo de amostras por extração em fase sólida}

Uma das técnicas mais utilizadas no preparo de amostras para análises cromatográficas é a extração em fase sólida (do inglês Solid Phase Extraction - SPE), técnica que permite não só a extração eficiente, mas também a pré-concentração dos analitos.

As etapas que envolvem a SPE estão descritas a seguir e representadas na Figura 2: Figura 2 - Procedimento de Extração em Fase Sólida

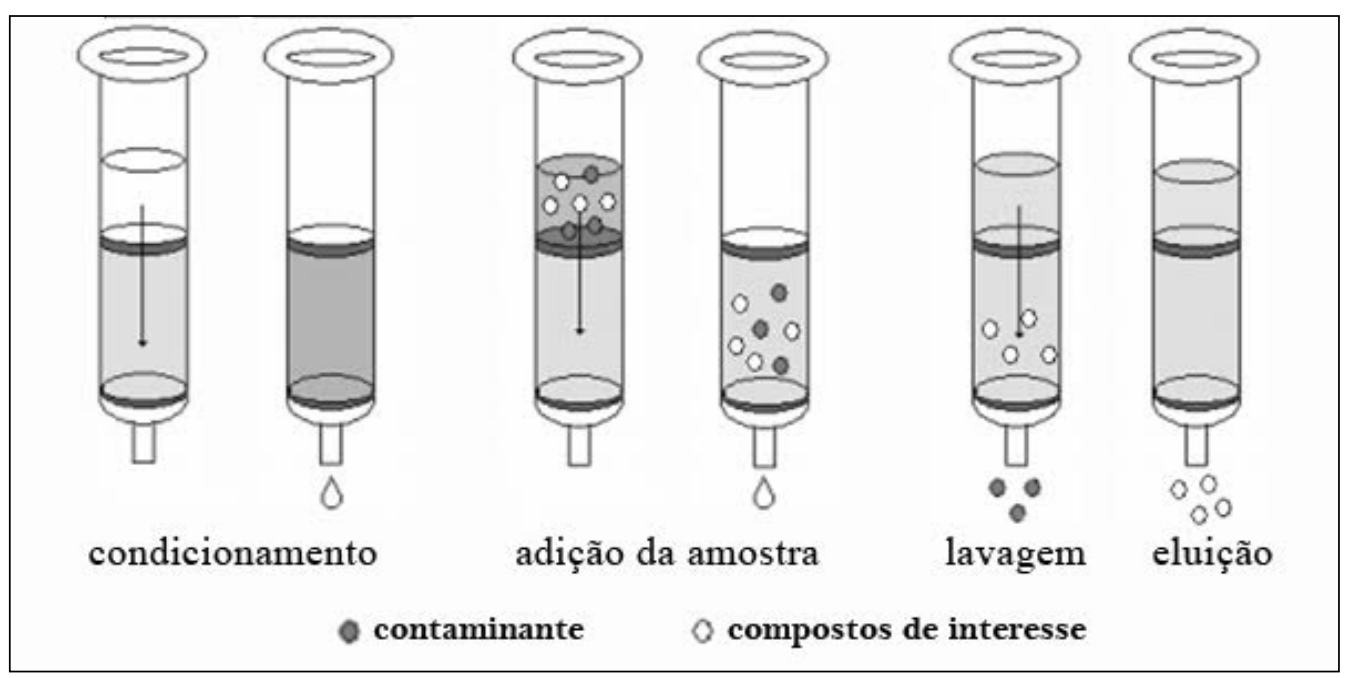

Fonte: Extração em Fase Sólida (SPE); Fernando Lanças (2004).

Ativação do sorvente com a passagem de um solvente apropriado para condicionar a superfície do sólido, como por exemplo metanol, e remoção do solvente de ativação com um líquido semelhante a amostra, neste caso, água ultrapura; 
Aplicação da amostra, quando os analitos ficarão retidos pelo sorvente;

Remoção de interferentes e da matriz residual com um solvente que não interaja com os analitos;

Eluição dos analitos com outro solvente apropriado

A separação e deteç̧ão dos analitos pode realizada com a utilização da Cromatografia Líquida (LC) e Cromatografia Gasosa (CG). $\mathrm{Na}$ técnica de LC, as substâncias são separadas por partição entre uma fase móvel líquida e uma superfície sólida, de acordo com a afinidade dos componentes pelas mesmas. A Cromatografia Líquida possui maior poder de resolução e velocidade de separação, permite a monitoração contínua do efluente da coluna e quantificação exata dos analitos. A coluna cromatográfica pode ser utilizada repetidas vezes de forma reprodutiva, com um sistema de automatização do procedimento analítico e do tratamento dos dados (NETO, 2003). O método possui alta sensibilidade, se adapta a espécies não-voláteis ou termicamente frágeis e se aplica a diversos campos de interesse para a ciência e para a indústria (SKOOG et al., 2008).

Outra técnica utilizada para análise de compostos em concentrações de até $\mu \mathrm{g} / \mathrm{L}$ é a Cromatografia Gasosa, onde a amostra é vaporizada no topo de uma coluna cromatográfica e a eluição é realizada através do arraste por um gás inerte. Diferentemente dos demais tipos de cromatografia, a fase móvel não interage com as moléculas do analito, servindo apenas como meio de transporte através da coluna. A coluna cromatográfica gasosa pode ser subdividida em cromatografia gás-sólido e cromatografia gás-líquido. A cromatografia gás-sólido baseia-se na adsorção das substâncias na fase gasosa em superfícies sólidas, e é útil para espécies de baixo peso molecular que não são retidas em colunas gás-líquido, como componentes do ar, sulfeto de hidrogênio, dissulfeto de carbono, óxidos de nitrogênio, monóxido de carbono, dióxido de carbono e gases raros. A cromatografia gás-líquido baseia-se na partição do analito entre a fase móvel gasosa e a fase estacionária líquida, imobilizada em um sólido inerte (SKOOG et al., 2008).

Tratando-se de detectores mais avançados, a utilização de espectrometria de massas pode ser usada para amostras que foram separadas pelo equipamento cromatográfico, seja a gás ou a líquido. Os espectrômetros de massas são muito úteis, tanto quando os espectros dos compostos já são conhecidos como quando se analisam compostos desconhecidos. No caso de compostos conhecidos, é realizada uma comparação do espectro do componente com uma biblioteca de espectro de massas que acompanha o software do equipamento. Quando se analisam compostos desconhecidos, características como o íon molecular, a sequência de fragmentação e outras evidências de espectrometria podem levar a identificação do composto (SILVERSTEIN; WEBSTER; KIEMLE, 2003).

Para a identificação com um espectrômetro de massas, o composto é ionizado e estes íons separados de acordo com sua razão massa/carga, e o número de íons 
correspondentes a cada unidade de massa/carga é registrado como um espectro (SILVERSTEIN; WEBSTER; KIEMLE, 2003).

O estudo de Batt, Kim e Aga (2007), utilizando o método de extração em fase sólida para a preparação de amostras de água residual, apresenta limites de detecção de $0,043 \mu \mathrm{g} / \mathrm{L}$ para a fluoroquinolona ciprofloxacina, $0,027 \mu \mathrm{g} / \mathrm{L}$ para sulfonamida sulfametoxazol e $0,052 \mu \mathrm{g} / \mathrm{L}$ para tetraciclina com sulfonamida, através da metodologia instrumental de LC-MS/MS.

Para amostras ambientais, o trabalho de Ibáñez et al. (2009) também utiliza a pré-concentração por SPE, aliada a análise de cromatografia líquida de ultraeficiência acoplada a espectrometria de massas por tempo de voo (do inglês Ultra-high-pressure liquid cromatography time-of-fight mass spectrometry - UHPLC-TOF-MS). Os limites de detecção obtidos pelos autores são expressos na Tabela 2.

Tabela 2 - Limites de deteç̧ão por UHPLC-TOF-MS para antibióticos em amostras ambientais

\begin{tabular}{l|l}
\hline Componente & Limite de Deteção $(\mu \mathrm{g} / \mathrm{L})$ \\
\hline Trimetoprima & 0,2 \\
\hline Lincomycin & 1 \\
\hline Ofloxacino & 0,4 \\
\hline Norfloxacino & 1 \\
\hline Cefaclor & 1 \\
\hline Ciprofloxacino & 1 \\
\hline Sulfametoxazol & 1 \\
\hline Moxifloxacin & 0,05 \\
\hline Azitromicina & 0,05 \\
\hline Clindamicina & 0,3 \\
\hline Eritromicina & 1 \\
\hline Claritromicina & 0,1 \\
\hline
\end{tabular}

Fonte: adaptado de Ibáñez et al. (2009).

\section{CONCLUSÃO}

A partir da revisão bibliográfica realizada, conclui-se que a área que engloba micropoluentes emergentes já possui uma grande demanda de estudos, principalmente no âmbito internacional. Técnicas de pesquisa avançada, através da pré-concentração com extração em fase sólida e com a utilização de equipamentos de cromatografia 
líquida e espectrometria de massas sofisticados são os mais utilizados para esta finalidade, por possuírem alta sensibilidade, detectando e quantificando componentes mesmo em concentrações mínimas. A conscientização da população também é um fator importante a se avaliar, pois o descarte indevido e a excreção de medicamentos, principalmente antibióticos, contribuem de forma significativa para o aumento dos níveis de poluentes emergentes. Estudos voltados à quantificação e aos potenciais mutagênicos e de genotoxicidade destas substâncias são necessários a fim de avaliar as consequências aos organismos consumidores, desta forma, torna-se primordial desenvolver tratamentos eficientes para a remoção dos mais diversos compostos e prever modificações dos parâmetros permitidos nas legislações atuais, assegurando as condições de potabilidade da água.

\section{REFERÊNCIAS}

AHUJA, Satinder (Ed). Handbook of water purity and quality. London: Academic, 2009.

ALVES, Célia. Tratamento de águas de abastecimento. 3. ed. Porto: Publindústria, 2010.

AQUINO NETO, Francisco Radler de; NUNES, Denise da Silva e Souza. Cromatografia: princípios básicos e técnicas afins. Rio de Janeiro: Interciência, 2003.

BATT, Angela L.; KIM Sungpyo; AGA Diana S. Comparison of the occureence of antibiotics in four full-scale wastewater treatment plants with varyngs designs and operations. Chemosphere, ELSEVIER, v. 68, p. 428-435, fev. 2007.

BILA, Daniele M.; DEZOTTI, Márcia. Fármacos no meio ambiente. Química Nova, Rio de Janeiro, v. 26, n. 4, p. 523-530, ju1/ago. 2003.

BILA, Daniele M.; DEZOTTI, Márcia. Desreguladores endócrinos no meio ambiente: efeitos e consequências. Química Nova, Rio de Janeiro, v. 30, n. 3, p. 651-666, fev. 2007.

BRASIL, Ministério da Saúde. Portaria n. o 2.914, de 12 de dezembro de 2011. Dispõe sobre normas de potabilidade de água para o consumo humano. Brasília: SVS, 2011.

DIAS, Débora X. Impacto ambiental e métodos de tratamento de micropoluentes para a minimização de efeitos nocivos ao ecossistema. Lorena, São Paulo. 46 f. Monografia (Graduação em Engenharia Bioquímica). 2012.

FENT, Karl; WESTON, Anna A.; CAMINADA, Daniel. Ecotoxicology of human pharmaceuticals. Aquatic Toxicololy, ELSEVIER, Muttenz, Switzerland, v. 76, p. 122-159, set. 2006.

FREIRE, Renato S.; et al. Novas tendências para o tratamento de resíduos industriais contendo espécies organocloradas. Química Nova, Campinas, SP, v. 23, n. 4, p. 504-511, jan. 2000.

GARRISON, A. W.; POPE, J. D.; ALLEN, F. R. Em Identification and Analysis of Organic Pollutants in Water; Keith, C. H., ed.; Ann Arbor Science Publishers: Ann Arbor, 1976, cap. 30. 
HIGNITE, C.; AZARNOFF, D. L. Drugs and drug metabolites as a environmental sewage water effluent. Life Sciences, Kansas,USA, v. 20, n. 2, p. 337-341, janeiro, 1977.

IBÁÑEZ, María et al. Screening of antibiotics in surface and wastewater samples by ultrahigh-pressure liquid chromatography coupled to hybrid quadrupole time-of-flight mass spectrometry. Journal of Chromatography A, ELSEVIER, Espanha, v. 1216, p. 2520-2539, jan. 2009.

KUNZ, A.; et al. Construção e otimização de um sistema para produção e aplicação de ozônio em escala de laboratório. Química Nova, Campinas, SP, v. 22, p. 425-428, 1999.

KUNZ, Airton; PERALTA-ZAMORA, Patrício; MORAES, Sandra G.; DURÁN, Nelson. Novas tendências no tratamento de efluentes têxteis. Química Nova, São Paulo, v. 25, n. 1, p. 78-82, jan/fev.2002.

LINDBERG, Richard et al. Determination of antibiotic substances in hospital sewage using solid phase extraction and liquid chromatography/mass spectrometry and group analogue internal standards. Chemosphere, ELSEVIER, Suécia, v. 57, p. 1479-1488, set. 2004.

MAGOSSI, Luiz Roberto; BONACELLA, Paulo Henrique. Poluição das águas. 2. ed. ref. São Paulo: Moderna, 2008.

MANFRED, C.; et al. Removal of antibiotics in conventional and advanced wastewater treatment: Implications for environmental discharge and wastewater recycling, Water Research, Vienna, Áustria, v. 39, p. 4797-4807, novembro, 2005.

COSTA NETO, Claudio. Análise orgânica: métodos e procedimentos para a caracterização de organoquímios. Rio de Janeiro: UFRJ, 2004.

MELO, Silene A. S.; et al. Degradação de fármacos residuais por processos oxidativos avançados. Química Nova, Araraquara, SP, v. 32, n. 1, p.188-197, dez. 2009.

RADJENOVIC, J.; PETROVIC, M.; BARCELÓ, D. Analysis of pharmaceuticals in wastewater and removal using a membrane bioreactor. Analytical and bioanalytical chemistry, Barcelona, Espanha, v. 387, p. 1365-1377, 2007.

REIS FILHO, Ricardo W.; et al. Fármacos, ETEs e corpos hídricos. Ambiente e Água, v. 2, n. 3, p. 54-61, 2007.

SILVERSTEIN, Robert M.; WEBSTER, Francis X.; KIEMLE, David J. Identificação espectrométrica de compostos orgânicos. 7. ed. Rio de Janeiro: LTC, 2013.

SKOOG, Douglas A.; HOLLER, F. James; NIEMAN, Timothy A. Princípios de análise instrumental. 5. ed. Porto Alegre: Bookman, 2008.

SOARES, Alexandra. F. S.; LEÃO, Mônica. M. D., Contaminação dos mananciais por micropoluentes e a precária remoção desses contaminantes nos tratamentos convencionais de água para potabilização. Revista Jurídica De Júri. ISSN 1809-8487, v. 14, n. 24, p. 36-85, jan./jun. 2015. Disponível em <https://aplicacao.mpmg.mp.br/xmlui/bitstream/ handle/123456789/1239/Contamina\%C3\%A7\%C3\%A3o\%20dos\%20mananciais. pdf?sequence=1>. Acesso em 08 junho 2017 . 
SOUZA, Carla P. F. A. de; FALQUETO Elda. Descarte de Medicamentos no Meio Ambiente no Brasil. Revista Brasileira de Farmácia, Rio de Janeiro, v. 96, n. 2, p. 1142-1158, 2015. Disponível em: <http://www.rbfarma.org.br/files/630--Descarte-deMedicamentos-no-Meio-Ambiente-no--Brasil---Formatado---1142-1158.pdf >. Acesso em: 30 mai.2017.

TAVARES, Walter. Antibióticos e quimioterápicos para o clínico. 2. ed. rev. atual. São Paulo: Atheneu, 2009.

TEDDER, William D.; POHLAND Frederick G. Emerging technologies in hazardous waste management III; American Chemical Society: Washington, 1993, cap. 5.

VERLICCHI et al. Hospital effluents as a source of emerging pollutants: An overview of micropollutants and sustainable treatment options. Journal of Hidrology. ELSEVIER, Ferrara, Itália, v. 389, p. 416-428. 2010. 